\title{
Calcitonin gene-related peptide facilitates sensitization of the vestibular nucleus in a rat model of chronic migraine
}

Yun Zhang ${ }^{1}$, Yixin Zhang ${ }^{1 *}$, Ke Tian², Yunfeng Wang ${ }^{1}$, Xiaoping Fan', Qi Pan ${ }^{1}$, Guangcheng Qin $^{3}$, Dunke Zhang ${ }^{3}$, Lixue Chen ${ }^{2}$ and Jiying Zhou'

\begin{abstract}
Background: Vestibular migraine has recently been recognized as a novel subtype of migraine. However, the mechanism that relate vestibular symptoms to migraine had not been well elucidated. Thus, the present study investigated vestibular dysfunction in a rat model of chronic migraine (CM), and to dissect potential mechanisms between migraine and vertigo.
\end{abstract}

Methods: Rats subjected to recurrent intermittent administration of nitroglycerin (NTG) were used as the CM model. Migraine- and vestibular-related behaviors were analyzed. Immunofluorescent analyses and quantitative realtime polymerase chain reaction were employed to detect expressions of c-fos and calcitonin gene-related peptide (CGRP) in the trigeminal nucleus caudalis (TNC) and vestibular nucleus (VN). Morphological changes of vestibular afferent terminals was determined under transmission electron microscopy. FluoroGold (FG) and CTB-555 were selected as retrograde tracers and injected into the VN and TNC, respectively. Lentiviral vectors comprising CGRP short hairpin RNA (LV-CGRP) was injected into the trigeminal ganglion.

Results: CM led to persistent thermal hyperalgesia, spontaneous facial pain, and prominent vestibular dysfunction, accompanied by the upregulation of c-fos labeling neurons and CGRP immunoreactivity in the TNC (c-fos: vehicle vs. $C M=2.9 \pm 0.6$ vs. $45.5 \pm 3.4 ;$ CGRP OD: vehicle vs. $C M=0.1 \pm 0.0$ vs. $0.2 \pm 0.0$ ) and VN (c-fos: vehicle vs. $C M=$ $2.3 \pm 0.8$ vs. $54.0 \pm 2.1$; CGRP mRNA: vehicle vs. $C M=1.0 \pm 0.1$ vs. $2.4 \pm 0.1)$. Furthermore, FG-positive neurons was accumulated in the superficial layer of the TNC, and the number of c-fost/FG+ neurons were significantly increased in rats with $C M$ compared to the vehicle group (vehicle vs. $C M=25.3 \pm 2.2$ vs. $83.9 \pm 3.0$ ). Meanwhile, $C T B-555+$ neurons dispersed throughout the VN. The structure of vestibular afferent terminals was less pronounced after CM compared with the peripheral vestibular dysfunction model. In vivo knockdown of CGRP in the trigeminal ganglion significantly reduced the number of c-fos labeling neurons (LV-CGRP vs. LV-NC $=9.9 \pm 3.0$ vs. $60.0 \pm 4.5$ ) and CGRP mRNA (LV-CGRP vs. LV-NC $=1.0 \pm 0.1$ vs. $2.1 \pm 0.2$ ) in the VN, further attenuating vestibular dysfunction after CM.

\footnotetext{
* Correspondence: Zhangyx_cq@163.com

'Department of Neurology, The First Affiliated Hospital of Chongqing

Medical University, 1st Youyi Road, Yuzhong District, Chongqing 400016,

China

Full list of author information is available at the end of the article
}

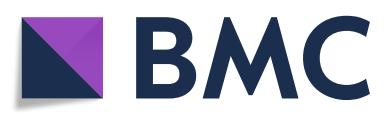

(- The Author(s). 2020 Open Access This article is licensed under a Creative Commons Attribution 4.0 International License, which permits use, sharing, adaptation, distribution and reproduction in any medium or format, as long as you give appropriate credit to the original author(s) and the source, provide a link to the Creative Commons licence, and indicate if changes were made. The images or other third party material in this article are included in the article's Creative Commons licence, unless indicated otherwise in a credit line to the material. If material is not included in the article's Creative Commons licence and your intended use is not permitted by statutory regulation or exceeds the permitted use, you will need to obtain permission directly from the copyright holder. To view a copy of this licence, visit http://creativecommons.org/licenses/by/4.0/ The Creative Commons Public Domain Dedication waiver (http://creativecommons.org/publicdomain/zero/1.0/) applies to the data made available in this article, unless otherwise stated in a credit line to the data. 
(Continued from previous page)

Conclusions: These data demonstrates the possibility of sensitization of vestibular nucleus neurons to impair vestibular function after CM, and anti-CGRP treatment to restore vestibular dysfunction in patients with CM.

Keywords: CGRP, Vestibular migraine, Trigeminal nucleus caudalis, Vestibular nucleus, Central sensitization, AntiCGRP treatment

\section{Introduction}

Migraine associated with recurrent vestibular symptoms are no strangers to clinicians. Migraine has been estimated to affect about $15 \%$ of the general population [1], and up to $85 \%$ patients with migraine suffers from balance problems and dizziness [2, 3]. Recently, International Headache Society and Bárány Society have prompted efforts to recognize and classify vestibular migraine (VM), which incorporates vestibular and migraine symptoms, as a novel subtype of migraine in International Headache Classification of Headache Disorders, 3rd edition [4]. Whether vestibular symptoms are associated symptoms as photophobia and phonophobia in migraine, or the distinctive entity that correlates with migraine are still in debate [5].

Migraine has been proposed as a complex sensory disorder with abnormal sensitization in central trigeminalvascular system $[6,7]$. Central sensitization of trigeminovascular neurons in trigeminal nucleus caudalis (TNC) has been considered to be the neural basis for sensory hypersensitivity in migraine, such as tactile allodynia [6]. We previously showed that the development of vertigo was lagged behind migraine by an average of 6 years in $\mathrm{VM}[7,8]$, and the prevalence of vertigo prominently increased in chronic migraine (CM) compared with episodic migraine [9]. Whether vestibular symptom is one of clinical manifestations of central sensitization for migraine remains unproven. Clinical data demonstrated that patients with VM exhibited abnormally elevated vestibularocular threshold [10], as well as reduced roll tilt threshold [11] compared to migraine patients without vestibular symptoms and patients with a peripheral inner-ear disorder, indicating that dysfunction of the vestibular nuclei (VN) might be an underlying mechanism for vestibular symptoms in VM. As an important sensorimotor center in brainstem, VN and its direct connection with TNC had been demonstrated in rodents [12]. Further, nociceptive trigeminal stimulation could induce vertigo in patients with migraine rather than controls [13]. Above data suggested that migraine-mediated sensitization of the TNC might affect the sensitivity of $\mathrm{VN}$ which receives TNC projections. However, whether vestibular sensitization is the potential mechanism for vestibular symptoms in migraine remains largely unknown.

The neuropeptide calcitonin gene-related peptide (CGRP) plays a crucial role in migraine based on its efficacy of anti-CGRP treatment in clinical trials against headache, and CGRP administration triggers delayed migraine-like headache in patients with migraine $[6,14]$. Pharmacological and electrophysiological studies demonstrated that CGRP might facilitate nociceptive signals in the spinal cord, rather than produce nociception, which contributes to the development of central sensitization [15]. Our previous study also found a significantly increased expression of CGRP in TNC in a rat model of CM, and that was parallel to the development of central sensitization [16]. Meanwhile, rodents exposed to repeated bouts of rotary stimulation had an increased number of CGRP expressing neurons in VN, whilst anisodamine could significantly decrease the CGRP expression in $\mathrm{VN}$ [17], pointing that CGRP might be a mediator in trigeminal-mediated sensitization of $\mathrm{VN}$.

Here our aim was to determine whether recurrent nitroglycerine (NTG) administration could induce vestibular dysfunction that is associated with migraine, and subsequently the effectiveness of CGRP knockdown to relieve these symptoms. We also wanted to determine central sensitization in TNC as a precursor in migraineassociated vestibular dysfunction. Using retrograde labeling of central trigeminocervical and vestibular neurons, we analyzed the functional connection changes caused by recurrent NTG administration and whether this manifested as a potential neural basis of VM in a preclinical model.

\section{Materials and methods \\ Animals}

Male Sprague Dawley rats (weighting 250-350 g) obtained from the Experimental Animal Center of Chongqing Medical University (Chongqing, China) were used in accordance with international standards under protocols approved by the Commission of Chongqing Medical University for ethics of experiments on animals. All studies were complied with the ARRIVE guidelines. All animals were randomly assigned to experimental groups. Animals were housed on a $12 \mathrm{~h}$ light-dark cycle with access to food and water ad libitum.

\section{Chronic migraine (CM) model}

The formula of NTG for injection was prepared as described previously $[16,18]$. In brief, a stock solution of $5.0 \mathrm{mg} / \mathrm{ml}$ NTG (Beijing Regent, China) was dissolved in 
$30 \%$ alcohol, 30\% propylene glycol, and distilled water. Prior to each administration, NTG was diluted to $1 \mathrm{mg} /$ $\mathrm{ml}$. Animals randomly received intraperitoneal administration of NTG $(2 \mathrm{ml} / \mathrm{kg})$ or vehicle ( $0.9 \%$ saline) every 2 days for 9 days [16]. According to previous study, 0.9\% saline was chosen as vehicle control due to the similar mechanical thresholds between $0.9 \%$ saline and $6 \%$ propylene glycol $+6 \%$ alcohol $+0.9 \%$ saline $[16]$.

\section{Peripheral vestibular dysfunction model}

Single trans-tympanic injection of kainic acid $(12.5 \mathrm{mM}$, $100 \mu \mathrm{L}$ ) was performed under anesthesia according to the procedures previously described $[19,20]$. Briefly, rats were anesthetized with $10 \%$ chloral hydrate $(4 \mathrm{ml} / \mathrm{kg}$, intraperitoneal) combined with analgesics $(0.01 \mathrm{mg} / \mathrm{kg}$ buprenorphine) as described previously [21], and then kept in lateral position. A sterile insulin syringe $(0.5 \times$ $16 \mathrm{~mm}$ ) was applied to penetrate the posterior portion of the tympanic membrane, and either kainic acid or $0.9 \%$ saline was injected into the right side of middle ear cavity slowly. After injection, the animal was kept in lateral position by resting on a electronical heating pad until recovered from the anesthesia.

\section{Stereotaxic surgery procedures and intracranial injection procedures}

Seven days prior to the first NTG or saline injection, rats were deeply anesthetized as described in Peripheral vestibular dysfunction model and placed on a stereotaxic frame for the injection of the retrograde tracer fluorogold (FG) or Cholera Toxin B (CTB) -555. At the end of the procedure, rats were treated as described in Peripheral vestibular dysfunction model for recovery.

A $2 \%$ solution of FG (Fluorochrome LLC, USA) was injected in bilateral caudal ventrolateral medullary regions following the coordinates as described in previous study (mediolateral: \pm 22.0 ; anteroposterior: -12.8 ; dorsoventral: - 10.0) [22]. For TNC injection, CTB-555 (C334776, Invitrogen) was positioned at $1-2.4 \mathrm{~mm}$ caudal to the obex and inserted into the bilateral caudal spinal trigeminal interpolaris ( $\mathrm{Sp5ic)}$, as lateral (about $2.7 \mathrm{~mm}$ ) as possible according to the previous studies [23, 24]. A volume of $1 \mu \mathrm{l}$ per site was slowly injected (over $30 \mathrm{~s}$ ) via Hamilton microsyringe $(10 \mu \mathrm{L})$, and the needle was left in place for additional 5 min when completed the injection.

\section{Lentiviral vectors preparation and trigeminal injection procedure}

The recombinant lentivirus containing the rat calca gene RNA interference (RNAi) sequence (sense: TGGAGC AGGAGGAGGAACA) was packaged using hU6-MCSUbiquitin-EGFP-IRES-puromycin vector by GeneChem (Shanghai, China). Blank lentivirus without RNAi sequence was prepared as control.
The lentivirus $(8 \times 105 \mathrm{TU}, 1 \mu \mathrm{L})$ was injected into the trigeminal ganglion as described previously [25]. After deeply anesthetized as described in Peripheral vestibular dysfunction model, rats were kept in lateral position on a heating pad. Facial hair between the ear and eye was shaved to expose the injection site (between the tympanic bulla, condylar process and angular process). The direction of injection was $90^{\circ}$ to the head midline and $15^{\circ}$ to the coronal plane. The injection depth of needle was $9 \mathrm{~mm}$. Rats randomly received either $10 \mu \mathrm{L}$ of viral vectors containing CGRP RNAi or blank viral vectors via microsyringe, and the needle was left in place for additional $5 \mathrm{~min}$ when completed the injection.

\section{Behavioral tests}

Behavioral assessments were performed by an investigator blinded to experiments as previously described [16]. Basal responses of thermal hyperalgesia was performed 15-20 min before each NTG injection. Post-treatment responses of thermal hyperalgesia, head grooming, balance beam walk, and negative geotaxis were performed at $2 \mathrm{~h}$ after each NTG administration. Vestibular dysfunction scores were evaluated at $2 \mathrm{~h}$ after the fifth NTG or single kainic acid injection [26-28]. Thermal hyperalgesia and head grooming were considered as indexes of spontaneous cutaneous cephalic and extracephalic allodynia [29]. Balance beam walk, negative geotaxis and vestibular dysfunction were a series of behavioral assessments for rodents' vestibular system [26-28, 30, 31].

\section{Thermal hyperalgesia}

Thermal sensitivity was tested using Hargreaves radiant heat apparatus (model PL-200, IITC, Taimeng, Chengdu, China) [21, 32]. Briefly, rats were accustomed in a smooth, glass-floored transparent cage (height: $15 \mathrm{~cm}$, width: $20 \mathrm{~cm}$ and length: $20 \mathrm{~cm}$ ) for $30 \mathrm{~min}$. Infrared radiation (intensity: 20) was then applied to the center of rats' hind paws. Withdrawal latency was automatically recorded when the hind paw moved. Thermal stimulation was stopped after $20 \mathrm{~s}$ in case of tissue damage. Each rat was tested three times with an interval of $5 \mathrm{~min}$ of each stimulation to calculate the average latency.

\section{Head grooming}

After each NTG injection, animals were placed in Plexiglas cages to habituate for $20 \mathrm{~min}$ as described previously [29, 33]. The time that animals spent on head grooming was recorded by a video camera, and the total recording time for per rat was $20 \mathrm{~min}$.

\section{Balance beam walk}

As described previously, a balance beam (length: $190 \mathrm{~cm}$, diameter: $2.5 \mathrm{~cm}$ ) was placed horizontally with an elevation of $40^{\circ}$, and a cushion was placed below to protect 
animals that fell [30]. A black plastic box $(13 \times 22 \mathrm{~cm}$ with a $5 \times 6 \mathrm{~cm}$ doorway) was set at the upper end of the rod to motivate the animal to cross the beam. Previous study demonstrated that rodents naturally sought out the darkness and run in an upwards direction [31]. The duration for traversing was recorded (3 trials per rat). Before each trial, animals were allowed to have a rest for $90 \mathrm{~s}$.

\section{Negative geotaxis}

Rats were placed onto a $40^{\circ}$ slope with their head downward as described previously [30), and the duration for a turn of $180^{\circ}$ was recorded. The maximum recording time was $20 \mathrm{~s}$ ( 3 trials per rat). The value of all three trials were averaged.

\section{Vestibular dysfunction scores}

As previously described, animal scores for vestibular functions evaluated the following four parameters: dyskinetic head movements, circling, retro-pulsion, tail-hang reflex, contact-inhibition reflex, and air-righting reflex [19, 26-28]. Each parameter was rated from 0 (normal behavior) to 2 (severe response). A maximum score of 12 was given with higher scores indicating worse performance.

\section{1) Dyskinetic head movements and circling}

These experiments contained two parts sharing same scoring system. Rats were placed individually in a transparent chamber $(50 \times 50 \mathrm{~cm})$, and were observed for head weaving or circling for a period of $2 \mathrm{~min}$. The response was rated as follows: $0=$ neither dyskinetic head movements nor circling, $1=$ sporadic dyskinetic head movements or circling, and $2=$ frequent to persistent dyskinetic head movements or circling.

\section{2) Retro-pulsion}

Rats were also placed individually in a transparent chamber as described above for an observation period for $2 \mathrm{~min}$. The response was rated as follows: $0=$ no backward steps; 1 = few backward steps; 2 = persistent backward walk.

\section{3) Tail-hang reflex}

Rats were lifted by the tail and the response was rated as follows: $0=$ straight body posture towards the ground with extension of forelimbs, $1=$ sporadic bending the body ventrally, and 2 = persistently bending the body towards its tail.

\section{4) Contact inhibition of righting reflex}

The rats were placed in a supine position while its back maintained contact with the table. Another horizontal surface was placed to touch its feet. The ability to go from a supine to a prone position was rated as follows: $0=$ completely righting, $1=$ partial righting, and $2=$ failed in righting.

\section{5) Air righting reflex}

The rats were held in a supine position and dropped from a height of $30 \mathrm{~cm}$ onto a foam cushion. The response was graded as follows: $0=$ completely righting and landing squarely on their feet, $1=$ partial righting or landing on side, and 2 = failed in righting and landing on back.

\section{Quantitative real-time polymerase chain reaction (qPCR)}

Based on previous studies, the rat TNC tissues, localized between -14 and $-16 \mathrm{~mm}$ from bregma, and the $\mathrm{VN}$ tissues, localized between -10 and $-12 \mathrm{~mm}$ from bregma, were extracted based on the rat brain atlas of Paxinos and Waston (6th edition) [16, 30]. Total RNA from tissues was extracted using RNAiso Plus reagent (TaKaRa, Dalian) (21). RNA concentration and purity were quantified spectrophotometrically with NanoDrop (Thermo, USA). First-strand cDNA was generated using1 mg of total RNA with reverse transcriptase PrimeScript ${ }^{\mathrm{m}}$ RT Reagent Kit (Takara, Dalian). The mRNA expression of rat CGRP was detected by qPCR withSYBR ${ }^{\circ}$ Premix Ex TaqTM II (TaKaRa, Dalian) with a CFX96 Touch thermocycler (BioRad, USA) according to the manufacturer's recommendation. The reaction mixture ( $20 \mu \mathrm{L}$ total) consisted of $10 \mu \mathrm{L}$ $2 \times \mathrm{SYBR}^{\triangleright} \mathrm{mix}, 8 \mu \mathrm{L}$ nuclease-free water, $0.5 \mu \mathrm{L}$ of primers (rat CGRP: F: 5'-GTTGGCTATTGTGCATCGTGTT-3', R: CCGCTTGAGGTTTAGCAGAGTTA-3'; GAPDH, F: 5'-ATGACTCTACCCACGGCAAGCT-3', R: 5'-GGAT GCAGGGATGATGTTCT-3') and $1 \mu \mathrm{L}$ diluted cDNA. The reactions were performed as follows: an initial 5 min's denaturation step, followed by 35 cycles of $95^{\circ} \mathrm{C}$ for $30 \mathrm{~s}$, $63^{\circ} \mathrm{C}$ for $30 \mathrm{~s}$, and $72^{\circ} \mathrm{C}$ for $45 \mathrm{~s}$, as well as a $7 \mathrm{~min}$ final extension step. Relative mRNA levels were calculated using the $\triangle \Delta \mathrm{Cq}$ method using GADPH mRNA as an internal control.

\section{Histological analysis}

Rats were deeply anesthetized with $10 \%$ chloral hydrate $(4 \mathrm{ml} / \mathrm{kg}$, intraperitoneal) and subcutaneously injected with $0.01 \mathrm{mg} / \mathrm{kg}$ buprenorphine, then transcardially perfused with heparinized $0.9 \%$ saline followed by $4 \%$ paraformaldehyde (PFA) in 0.1 M PBS (pH 7.4) (for immunofluorescence staining) or $2.5 \%$ glutaraldehyde (for transmission electron microscopy). 


\section{Immunofluorescence staining}

Immunofluorescence staining was performed on fixed frozen brain sections as previously reported [16]. Brains were post-fixed in $4 \%$ PFA overnight at $4{ }^{\circ} \mathrm{C}$, and cryoprotected at $4{ }^{\circ} \mathrm{C}$ by successively immersed in a gradually increased concentration of sucrose solution $(20 \%$ to $30 \%)$ until the tissues sank to the bottom. The transverse and sagittal sections of the $\mathrm{VN}$, as well as the transverse sections of TNC were cut at $10 \mu \mathrm{m}$ thickness on cryostat (Leica, Japan). Sections were permeabilized with $0.3 \%$ Triton X-100 (Beyotime, China) for $10 \mathrm{~min}$ at $37^{\circ} \mathrm{C}$, then blocked in 10\% normal goat serum (Boster, China) in PBS for $30 \mathrm{~min}$. After washing with PBS for three times ( 5 min each), brain sections were incubated at $4{ }^{\circ} \mathrm{C}$ overnight with the following primary antibodies: antiCGRP (1:100, sc-57,053, Santa Cruz, USA); anti-c-fos (1: 1000, 226,003, SYnaptic Systems, Germany); anti-NeuN (1:100, ab104224, Abcam, UK); anti-Glutamate (1:100, G9282, Sigma, USA); anti-Glutamic Acid Decarboxylase 65/67 (GAD67, 1:100, G5163, Sigma, USA). Sections were rinsed three times with PBS, followed by a 90 min's incubation at $37{ }^{\circ} \mathrm{C}$ with secondary antibodies: Alexa Fluor 488-conjugated goat anti-rabbit immunoglobulin G (IgG, 1:200, Beyotime, China), Alexa Fluor 488conjugated goat anti-mouse IgG (1:200, Beyotime, China), and Cy3-conjugated goat anti-mouse IgG (1:200, Beyotime, China), and then washed again with PBS for three times. Finally, slides were covered with DAPI (Beyotime, China). The sections were imaged under a fluorescence confocal microscope (ZEISS, Germany).

Sections were examined and images were acquired with a ZEISS Axio Imager A2 microscope equipped with structured illumination (Zen). Morphological identification of TNC was determined as previously described [16, 21]. The VN was identified under light microscopy through Paxinos and Watson's stereotaxic atlas. C-fos+, CGRP+, and FG+ cells were quantified on both sides for TNC and VN from selected serial transverse sections collected from the rostral to caudal part of the brainstem. The number of immunolabeled cells was counted through the optical fractionator method under a 20x objective [34]. Each rat was collected six sections from the TNC and another six sections from the $\mathrm{VN}$ for cell counting, and the results were averaged and expressed as cells/field of view. To avoid counting the same neuron twice, each section was separated by at least $250 \mu \mathrm{m}$ [22]. For each labeling in the TNC and VN, two quantified images were randomly selected with at least $480 \mu \mathrm{m}$ apart [35]. Considering that CGRP exclusively expressed on the terminals of primary afferent fibers in the TNC, the mean optical density (OD) of CGRP were used to estimate the fluorescence intensity of CGRP over a microscopic field of $20 \times$. Two investigators were counted individually, and both of them were blinded to the groups.
The proportion of FG-filled TNC and CTB-555-filled neurons that also contained c-fos labeling was analyzed as described above. FG-positive/c-fos-positive and FGpositive/c-fos-negative cells were counted at $\times 20$ magnification. The OD of CTB-555 in the $\mathrm{VN}$ were analyzed under $\times 10$ magnification.

\section{Transmission electron microscopy}

Tissue preparation for transmission electron microscopy was performed as previously described $[19,36]$. In brief, Inner ears were rapidly removed, followed by unfolding acoustic vesicle to expose cochlea and its surrounding structures. Whole vestibular organs were fixed by $4 \%$ glutaraldehyde with phosphate buffer $(0.1 \mathrm{M}, \mathrm{PH} 7.2)$ at $4{ }^{\circ} \mathrm{C}$ overnight. Then, vestibular organs were decalcified in $10 \%$ EDTA for $14 \mathrm{~d}$ at room temperature. After being rinsed with phosphate buffer for $30 \mathrm{~min}$, the samples were post-fixed with $1 \%$ osmium tetroxide (OsO4) for $1.5 \mathrm{~h}$, followed by staining with aqueous uranyl acetate for $1 \mathrm{~h}$, dehydrating in a graded series of ethanol and embedding in Epon-Araldite resin (Canemco \& Marivac, Lakefield, Quebec, Canada). Ultrathin sections (silvergold, 80-90 nm) were cut using an ultramicrotome (Reichert-Jung, Inc., Cambridge, UK), and sections were counterstained with $0.3 \%$ lead citrate, and visualized on a transmission electron microscope (EM420, Koninklijke Philips Electronics N.V., Amsterdam, The Netherlands).

Cells were classified based on morphological criteria in accordance with previous protocol $[19,20]$. The proportion of hair cells contacted by their swollen terminals (width $>1 \mu \mathrm{m}$ ) were summed on 3 ultrathin sections collected from 3 different sets from striolar and extrastriolar regions. These images were analyzed using Imagepro Plus 6.2 software (Bethesda, MD, USA).

\section{Statistical analysis}

Data are presented as mean \pm SEM. All statistical analyses were performed by SPSS software (SPSS Inc., IBM, USA). Student's $t$-test was used for statistical comparisons between two groups. One-way or two-way ANOVA followed by post hoc analysis with the Tukey test was used for statistical comparisons among groups. MannWhitney $U$ test was selected for the nonparametric analysis. Results were defined significant at $p<0.05$.

\section{Results}

Recurrent NTG injection induced hyperalgesia and vestibular dysfunction

Consistent with our previous studies [37], we observed that in rats treated with NTG, the paw withdrawal latencies to noxious heat were markedly decreased in a time dependent manner on day 5, 7, and 9 as compared to vehicle control group (Fig. 1b). Chronic injection of NTG produced progressive basal hypersensitivity (Fig. 1b) and 


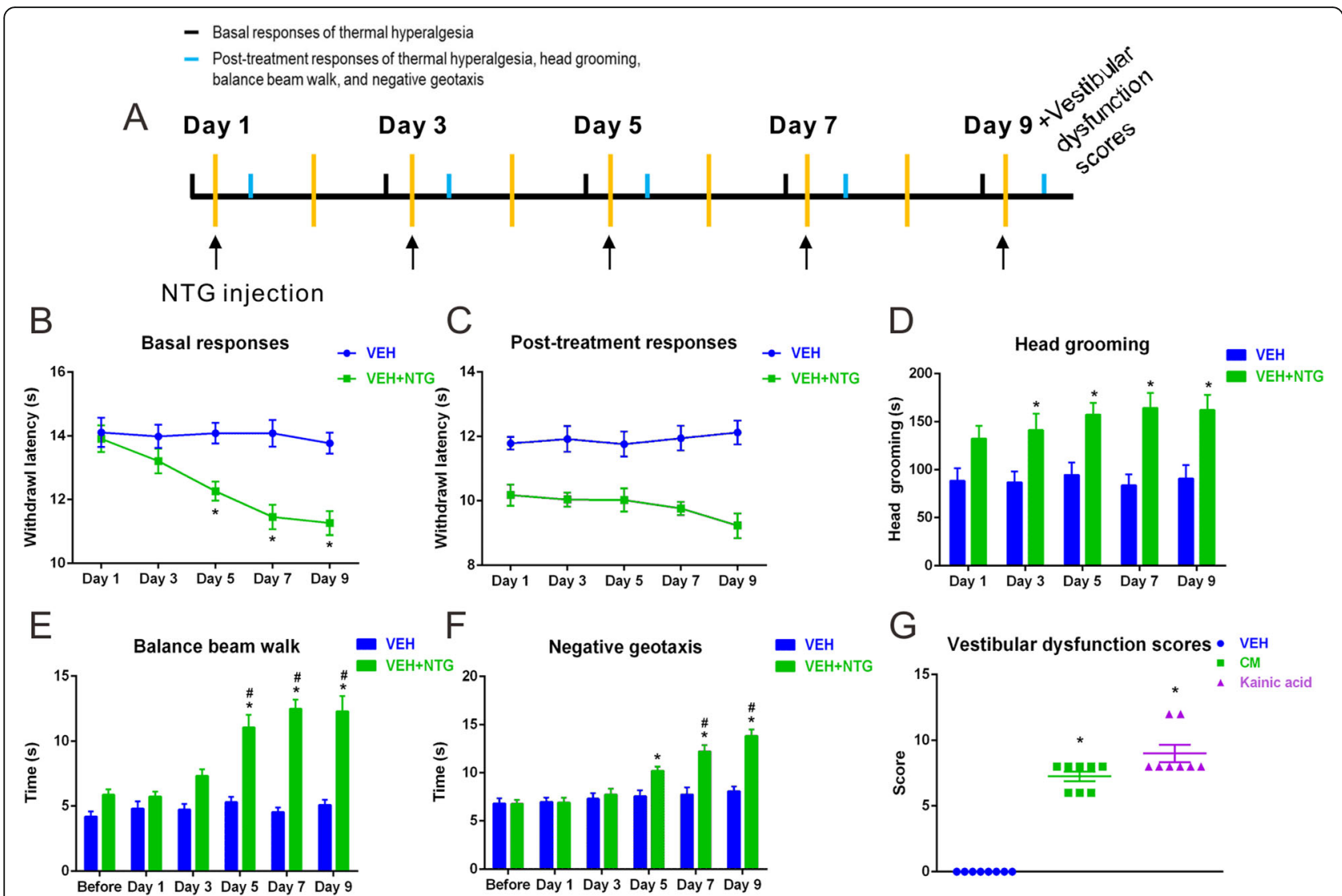

Fig. 1 Recurrent nitroglycerine (NTG) injection induced thermal hyperalgesia and vestibular dysfunction. a Timeline of behavior studies' protocol. b Basal and post-treatment responses (c) of hindpaw thermal hyperalgesia were markedly increased in a time dependent manner after NTG injection. $\mathbf{d}$ Head grooming time was significantly increased in NTG-treated rats compared with vehicle-treated group. Balance beam walk (e), geotaxis reflex (f) and vestibular dysfunction scores $(\mathbf{g})$ showing that repeated NTG administration produced significant impairments in dynamic and static postural control compared with the vehicle group. $n=8$ /group. Data are mean \pm SEM. Analysis of variance (ANOVA), Tukey; ${ }^{*} p<0.05$ compared with vehicle, \#p $<0.05$ compared with before NTG injection

acute allodynia (Fig. 1c). We also found that NTG significantly increased head grooming time on day $3,5,7$, and 9 as compared to vehicle control group (Fig. 1d).

We then asked whether chronic intermittent injection of NTG exacerbated vestibular functions. The alternation of vestibular function was determined by a series of behavior studies: balance beam and negative geotaxis for the assessment of dynamic postural changes during vestibulospinal reflexes; vestibular dysfunction scores for the evaluation of rodents' static postural control [26-28]. NTG injection extended the time that rats spent traversing the balance beam, reaching significantly on day 5,7 , and 9 as compared to the vehicle and before injection data (Fig. 1e). On day 9, NTG-received rats needed approximately triple time to traverse the balance beam as compared to the vehicle (Fig. 1e). Similar results were observed in negative geotaxis test. NTG-received rats spent significantly more time to turn to $180^{\circ}$ upward as compared to the vehicle on day 5, 7, and 9 (Fig. 1f). Furthermore, the severity of static vestibular dysfunction was significantly increased in NTG-received rats compared with the vehicle (Fig. 1g). Behavior studies revealed that the development of vestibular dysfunction was in parallel with that of pain hypersensitivity after CM.

\section{Morphology of vestibular afferent terminals was preserved after CM}

Among patients with migraine, up to $25 \%$ prevalence of peripheral vestibular abnormities has been reported, including peripheral nystagmus and unilateral hearing loss $[38,39]$. In the present study, vestibular dysfunction results showed that the severity of CM-induced vestibular dysfunction was comparable with the kainic acid treated group (Fig. 1g). Thus, researchers postulated that the damage of vestibular afferent terminals caused by migraine-associated vasospasm might mediate vestibular symptoms [40-42]. To investigate whether the CMinduced vestibular dysfunction stem from direct damage to vestibular apparatus, we used electron microscopy to examine the percentage of type I hair cells displaying 
swollen terminals in rat right utricles $2 \mathrm{~h}$ post CM. Since the severity of vestibular dysfunction and glutamateelicited tissue damage peaked at $2 \mathrm{~h}$ post injection, morphological evaluation at subcellular level were determined at $2 \mathrm{~h}$ post kainic acid injection [19].

In vehicle-treated group, the typical morphology of sensory epithelia, that supporting cells were in order and type I hair cells were like pear shape surrounded by calyx terminals, was observed (Fig. 2). CM group's supporting cells were still present in order, but hair cells were partially separated by few vacuoles (Fig. 2). Following kainic acid injection, supporting cells were not in order and the type of hair cells became undetermined that the afferent terminals were highly swollen leaving large vacuoles among the sensory epithelia (Fig. 2). The population of hair cells displaying swollen terminals in kainic acid-treated group $(71.9 \pm 4.1 \%)$ was significantly higher $(p<0.05)$ than those observed in vehicle $(5.1 \pm$ $1.4 \%)$ and $\mathrm{CM}$ group $(9.2 \pm 1.3 \%)$, while the proportion was comparable between vehicle and CM group. Preserved morphology of vestibular end-organs in CM group indicated that $\mathrm{CM}$-induced vestibular dysfunction might likely to be central origin.

\section{Elevation of c-fos and CGRP in the TNC and VN after CM}

$\mathrm{C}$-fos has been considered to be a marker for the neuronal activation after noxious stimulation, and its upregulation underly the sustained mechanical hypersensitivity in CM [37, 43]. Immunofluorescence staining showed that the number of c-fos positive cells in the TNC was significantly increased post $\mathrm{CM}$ (vehicle vs. $\mathrm{CM}=2.9 \pm$ 0.6 vs. $45.5 \pm 3.4$, Fig. 3a and b). Given the key position of $\mathrm{VN}$ in vestibulospinal reflexes and posture control [30], we next sought to examine the changes of $\mathrm{VN}$ neurons after CM. Accordingly, c-fos immunoreactivity was also significantly increased in the $\mathrm{VN}$ post $\mathrm{CM}$ (vehicle vs. $\mathrm{CM}=2.3 \pm 0.8$ vs. $54.0 \pm 2.1$, Fig. $3 \mathrm{a}$ and b). No evident difference was detected in the overall number of cfos positive cells between right and left side of the TNC and VN (Fig. 3b).

Moreover, CGRP emerges as a critical endogenous mediator of migraine, especially in the development and maintenance of central sensitization [37, 44]. The density of CGRP-immunoreactive fibers in the TNC was significantly increased in the superficial layers of TNC after $\mathrm{CM}$ (vehicle vs. $\mathrm{CM}=0.1 \pm 0.0$ vs. $0.2 \pm 0.0$, Fig. $3 \mathrm{c}$ and d). We further suspected whether endogenous CGRP expression in the VN would be changed after NTG administration. We analyzed CGRP expression in the $\mathrm{VN}$ by qPCR after CM. The mRNA level of CGRP was significantly increased after $\mathrm{CM}$ relative to vehicle-treated group (Fig. 3e). Although CGRP positive cells were widely distributed throughout the four major vestibular nucleus as defined in the rat brain atlas of Paxinos and Watson, we found that CGRP immunoreactivity was moderately higher in the medial vestibular nucleus compared to the superior, lateral and spinal nucleus after CM (Fig. 3c).

\section{TNC-projecting VN neurons were activated after CM}

To determine whether trigeminovestibular neurons are activated by $\mathrm{CM}$, we evaluated $\mathrm{c}$-fos+/FG+ neurons in the TNC, as well as CTB-555+ neurons in the VN after CM. Retrogradely transported FG was accumulated

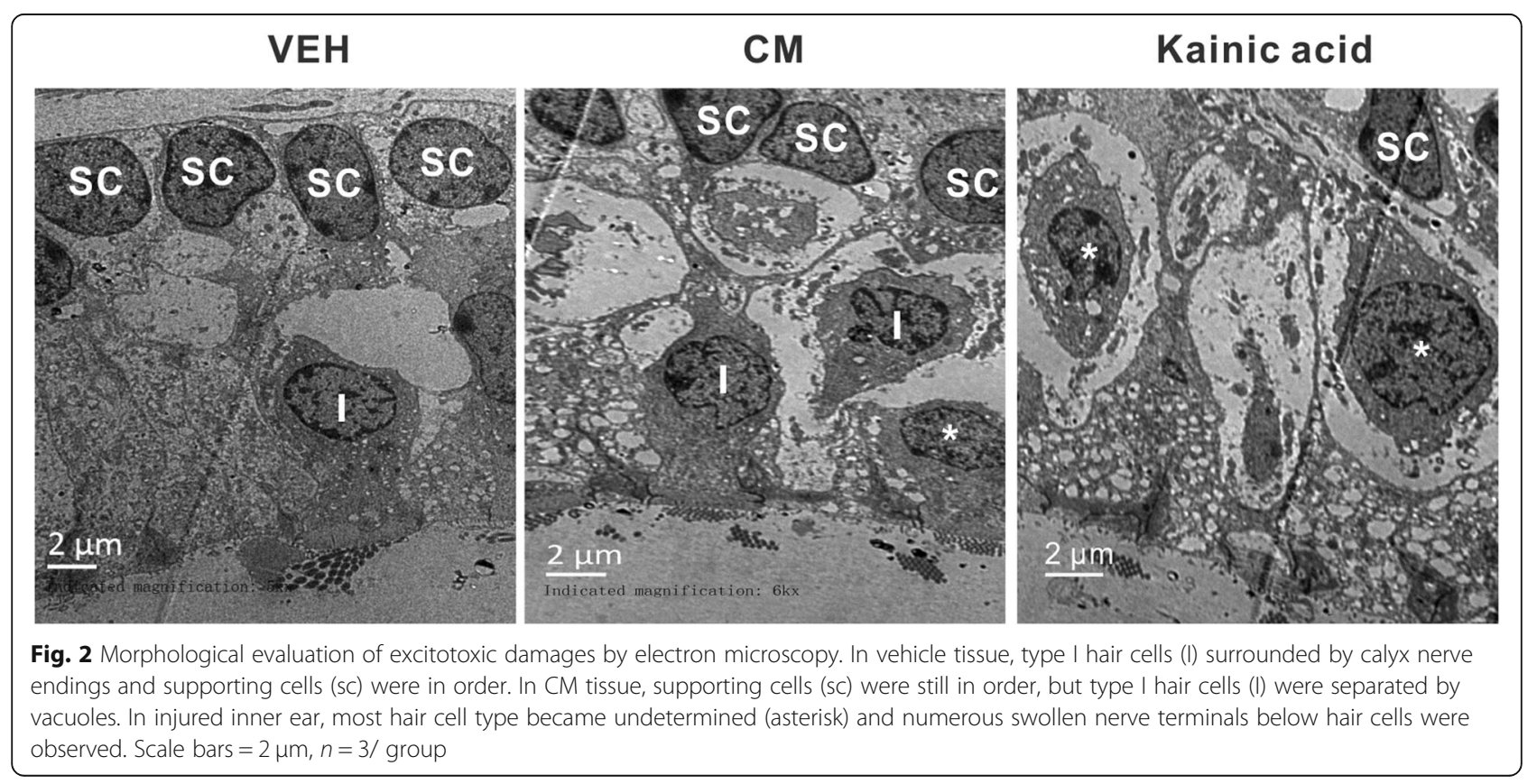




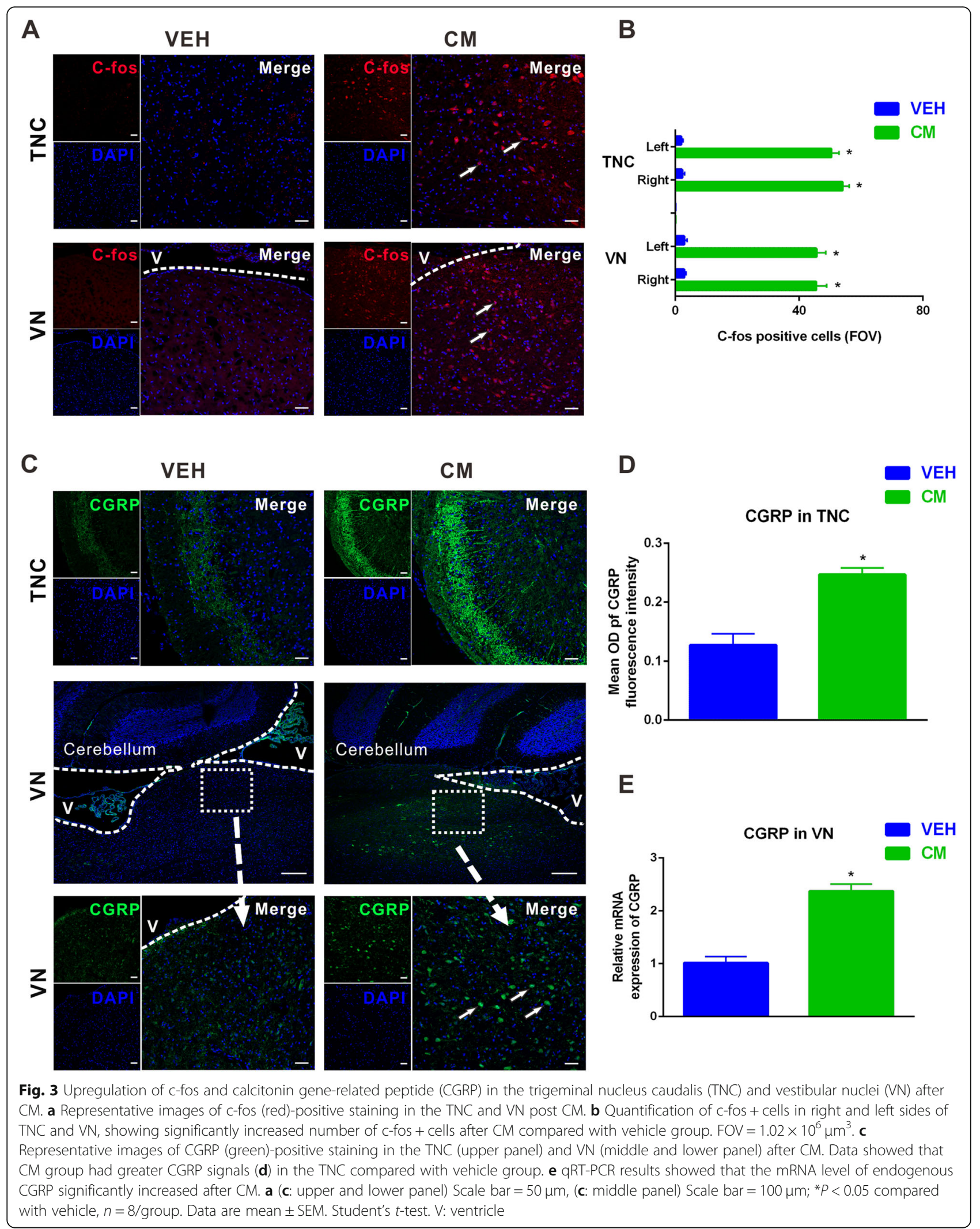


under physiological condition predominantly in the superficial layer of the TNC (Fig. 4a). Compared to the vehicle group, the number of FG+ neurons increased significantly in the bilateral TNC after CM (Fig. 4a and b). We also found that the number of c-fos $/$ FG+ neurons were significantly increased in rats with CM compared to the vehicle group (vehicle vs. $\mathrm{CM}=25.3 \pm 2.2$ vs. $83.9 \pm 3.0$ ), revealing that $\mathrm{FG}+$ neurons in the TNC were primarily CM-activated neurons (Fig. 4a-c). In the meantime, CTB$555+$ neurons dispersed throughout the VN (Fig. 4d).
There was a slight increase in CTB-555 immunoreactivity in the $\mathrm{CM}$ group compared to vehicle group, which was not reach statistically difference (Fig. 4d-e).

We further sought to localize the amino acid in the cells that were both activated after $\mathrm{CM}$ and had direct projections to the $\mathrm{VN}$. We performed immunofluorescence concomitantly for FG, glutamate and GABA. CMactivated TNC neurons with projections to $\mathrm{VN}$ were either glutamate- or GABA- immunofluorescent (Fig. 4f and g), suggesting both of excitatory and inhibitory input

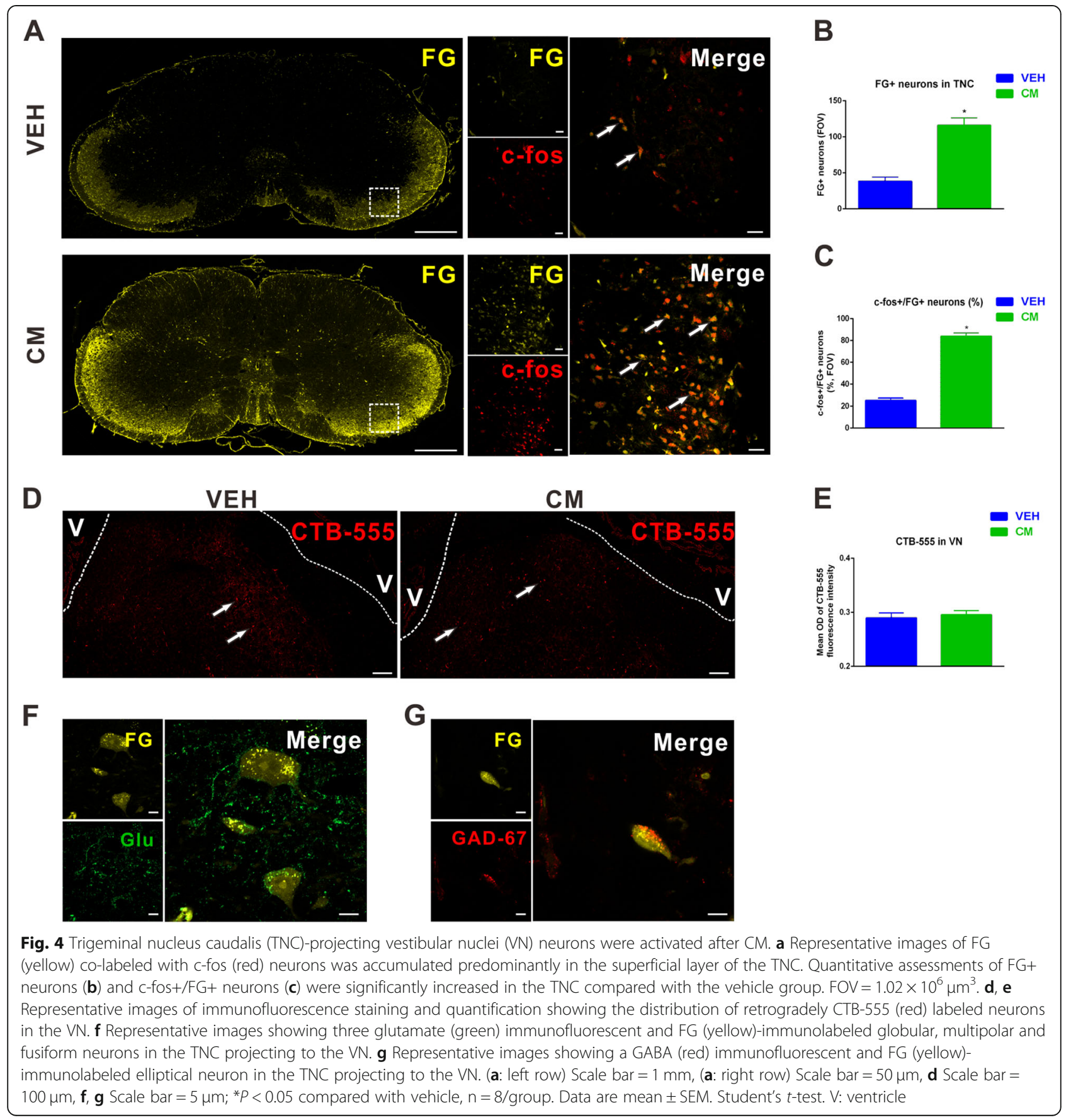


to these trigeminovestibular neurons. Aside from neurotransmitter content, the glutamate-immunofluorescent activated projection neurons were cytologically indistinguishable from the GABA immunofluorescent subpopulation (Fig. $4 \mathrm{f}$ and g).

\section{Knockdown of CGRP in the trigeminal ganglion reduced c-fos and CGRP levels in the TNC and VN after CM}

In vivo studies suggest that CGRP is synthetized by small- and medium-diameter neurons in the trigeminal ganglion and released from their central terminals in the brainstem to produce the central sensitization in $\mathrm{CM}$ [15]. To investigate the effects of CGRP knockdown on neuronal activation in the VN after $\mathrm{CM}$, we injected lentiviral vectors comprising CGRP short hairpin RNA (LVCGRP) or blank lentiviral vectors (LV-NC) with enhanced green fluorescence protein (GFP) into bilateral trigeminal ganglion 2 weeks before the initial NTG administration. Fluorescently labeled neurons were abundantly accumulated in the V1 and V2 branches of the trigeminal ganglion (Fig. 5a).

Compared with LV-NC, pretreatment of LV-CGRP in the trigeminal ganglion markedly attenuated CGRP immunoreactivity in the TNC after CM (Fig. 5b and d). The addition of LV-CGRP after CM significantly reduced CGRP mRNA levels in the VN (LV-CGRP vs. LV$\mathrm{NC}=1.0 \pm 0.1$ vs. $2.1 \pm 0.2$, Fig. $5 \mathrm{c}$ and e). In the meanwhile, c-fos immunoreactivity in the TNC (LV-CGRP vs. LV-NC $=22.4 \pm 3.2$ vs. $63.6 \pm 5.1$ ) and VN (LV-CGRP vs. $\mathrm{LV}-\mathrm{NC}=9.9 \pm 3.0$ vs. $60.0 \pm 4.5)$ were both significantly reduced in the $\mathrm{CM}+\mathrm{LV}$-CGRP group compared to $\mathrm{CM}+\mathrm{LV}-\mathrm{NC}$ group (Fig. 5g-h).

\section{Knockdown of CGRP in the trigeminal ganglion ameliorated hyperalgesia and vestibular dysfunction after CM}

To further clarify the effects of CGRP knockdown on CM-induced hyperalgesia and vestibular dysfunction, a series of behavior studies were assessed. The basal and post-treatment thermal thresholds were significantly increased in LV-CGRP-treated group compared with those in the LV-NC group (Fig. 6a and b), and significant changes were observed on day 7 , and on day 9. LVCGRP-treated rats spent less time on head grooming compared to the LV-NC group since day 3, indicating a markable improvement on spontaneous facial pain (Fig. 6c). In the balance beam walk and negative geotaxis test, LV-CGRP-treated animals spent less time on traversing beam and flipping to the prone position (Fig. $6 \mathrm{~d}$ and e). Moreover, LV-CGRP-treated rats performed significantly better in dynamic postural controls compared with LVNC group, as demonstrated by the less vestibular dysfunction score (Fig. 6f). Collectively, knockdown of CGRP in the trigeminal ganglion attenuated neuronal activation and CGRP translation in the $\mathrm{VN}$ and restored vestibular function after $\mathrm{CM}$, indicating a key role of CGRP in the development of vestibular dysfunction in CM.

\section{Discussion}

Vestibular symptoms are prevalent among patients with migraine $[2,3]$. The mechanism that relate vestibular symptoms to migraine had not been well elucidated. Thus, current therapies are based on experts' experience and little clinical progress has been made to develop therapeutic strategies targeting this subpopulation. Central sensitization has been demonstrated as a primary pathophysiological process after CM, in which CGRP has an essential role [6]. To date, clinical trials witness great success in anti-CGRP treatment on migraine headache [45]. Therefore, whether sensitization in the vestibular nucleus attribute to the development of vestibular dysfunction after CM, and whether antiCGRP treatment could attenuate vestibular symptoms in migraine needs further illustrating.

The present study revealed that the development of vestibular dysfunction coincide with that of hyperalgesia in a rat model of CM. Meanwhile, the structure of vestibular afferent terminals was preserved after CM. CM induced neuronal activation in the $\mathrm{TNC}$ and $\mathrm{VN}$, and $\mathrm{CM}$-activated neurons in the TNC were primarily TNCprojecting VN neurons. In vivo knockdown of CGRP in the trigeminal ganglion could alleviate neuronal activation and upregulation of CGRP in the $\mathrm{VN}$, further attenuating vestibular dysfunction in CM rats. Collectively, these studies suggested the possibility of vestibular sensitization to impair vestibular function after $\mathrm{CM}$, and anti-CGRP treatment to restore vestibular dysfunction in patients with CM.

Systemic recurrent NTG administration has been proven to be a reliable method to produce the preclinical CM model: inducing acute and sustained hypersensitivity, which mimics the core clinical characteristics of $\mathrm{CM}$; causing distinctively associated features, like photophobia, facial grimace behaviors, and upregulation of CGRP in the TNC and dura mater $[15,45]$. However, little is known about the changes of vestibular-mediated behaviors in this CM model. Further, since up to one-fourth percentage of peripheral vestibular deficits, like hearing loss and endolymphatic hydrops, has been detected among migraine patients with vestibular symptoms and the development of vestibular symptoms often lagged several years behind headache $[8,46]$, some researchers deduce that the vestibular dysfunction in migraine patients may result from the damage of peripheral vestibular apparatus [40-42, 47]. In the present study, we first revealed that recurrent NTG administration produced allodynia and significant vestibular dysfunction, and the 
A
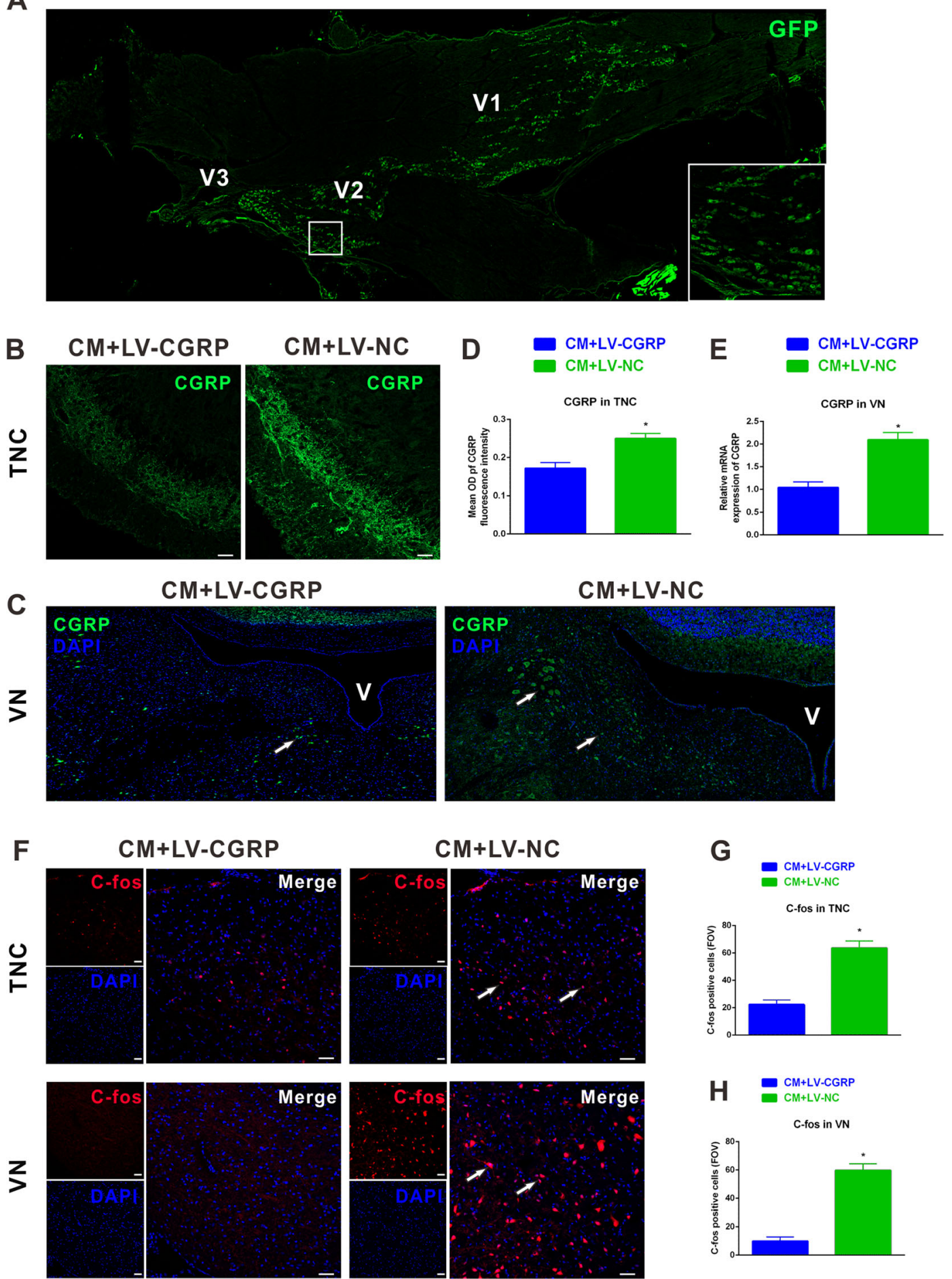

Fig. 5 Knockdown of calcitonin gene-related peptide (CGRP) in the trigeminal ganglion reduced c-fos and CGRP levels in the trigeminal nucleus caudalis (TNC) and vestibular nuclei (VN) after CM. a Representative images of immunofluorescence staining showing GFP (green) labeled lentivirus was injected into the trigeminal ganglion and dispersed in V1 and V2 branches. Representative images of immunofluorescence staining showing the immunoreactivity of CGRP in the TNC (b) and VN (c). Quantitative assessments showed that pretreatment of lentiviral vectors comprising CGRP short hairpin RNA (LV-CGRP) significantly reduced CGRP immunoreactivity in the TNC (d) and mRNA levels of CGRP in the VN (e). $\mathbf{f}$ Representative images showing the c-fos + neurons in the TNC and VN. (g) In the LV-CGRP treated group, the number of c-fos positively stained neurons in the TNC and VN significantly reduced compared with blank lentiviral vectors (LV-NC). FOV $=1.02 \times 10^{6} \mu \mathrm{m}^{3}$. $\mathbf{b}, \mathbf{f} \mathrm{Scale}$ bar $=$ $50 \mu \mathrm{m} ;{ }^{*} P<0.05$ compared with vehicle, $\mathrm{n}=8$ /group. Data are mean \pm SEM. Student's $t$-test 


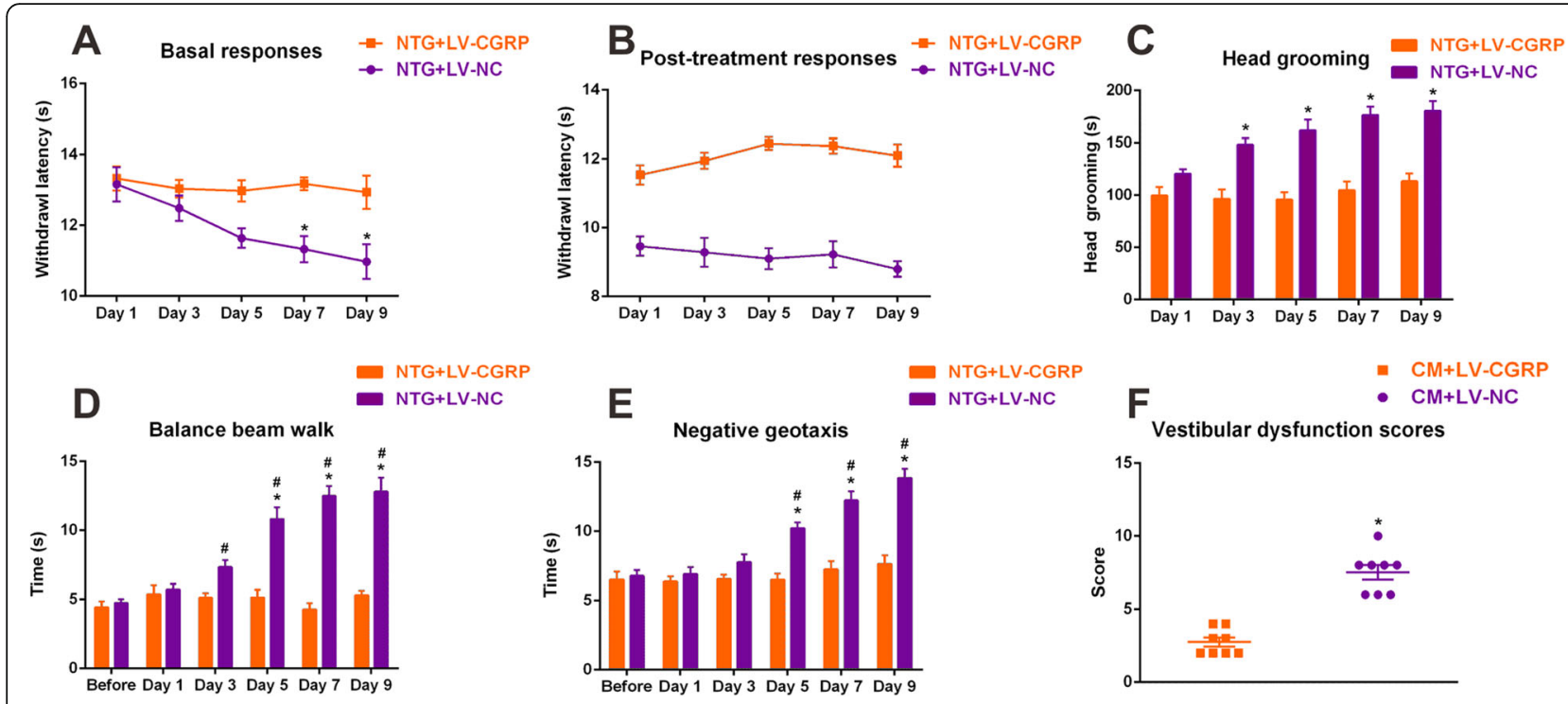

Fig. 6 Knockdown of calcitonin gene-related peptide (CGRP) in the trigeminal ganglion ameliorated hyperalgesia and vestibular dysfunction after CM. a Basal and post-treatment responses (b) of hindpaw thermal hyperalgesia were significantly decreased with pretreatment of LV-VGRP. (c) Head grooming time was significantly decreased in LV-CGRP-treated rats compared with LV-NC-treated group. Balance beam walk (d), geotaxis reflex (e) and vestibular dysfunction scores ( $\mathbf{f}$ ) showing that pretreatment of LV-CGRP restored impairments in dynamic and static postural control compared with the LV-NC group. $n=8 /$ group. Data are mean \pm SEM. Analysis of variance (ANOVA), Tukey; ${ }^{*} p<0.05$ compared with vehicle, \#p< 0.05 compared with before NTG injection

severity of vestibular dysfunction was comparable with kainic acid treated group. The development of vestibular dysfunction in our study is in line with clinical-based studies showing that most migraine patients are vestibular symptoms-free during the initial years of their migraine experience, but gradually develop motion intolerance or vestibular symptoms with increasing frequency of migraine attacks [9]. Meanwhile, morphological examination of the afferent terminal impairments showed that the swelling and structure disorders were much less pronounced in the CM group when compared with kainic acid treated group. These data indicated that it might be more likely a central, rather peripheral, component contribute to the vestibular dysfunction in migraine.

Migraine has been more generally considered as a neurological disorder of sensitization, with heightened sensitivity to light, sound, smell and motion [48]. Clinical and preclinical studies show that the central sensory systems are sensitized in migraine [49]. Thus, a reasonable hypothesis is that sensitization of vestibular pathways may contribute to the enhanced motion sickness susceptibility and episodic attacks of vestibular symptoms in migraine [50]. C-Fos has been widely used as an early indicator of neuronal activation that normally responds within $2 \mathrm{~h}$ [51]. Long-term c-Fos expression after NTG administration suggests continuous activation of secondary sensory neurons $[37,52]$. In our study, significant fos-protein labeling in the superficial lamina of the
TNC and VN after chronic NTG administration were observed. It is noteworthy that anatomic pathways giving extensive projections between brainstem regions associated with the TNC and the VN have been traced in the physiological condition [12]. We then asked whether $\mathrm{CM}$-activated neurons belonged to the population of trigeminovestibular neurons, and whether persistent activation of TNC neurons facilitated subsequent activation of VN neurons. To gain a further understanding, FluoroGold and CTB-555 were selected as retrograde tracers in vehicle and $\mathrm{CM}$ groups, mainly based on published reports of their sensitivity and uni-directional transportation [22, 53]. Our immunofluorescent results showed that FluoroGold labeling neurons were predominantly located in the superlayer of the TNC, and CM-activated neurons in the $\mathrm{TNC}$ were primarily $\mathrm{TNC}$-projecting $\mathrm{VN}$ neurons. Furthermore, to investigate whether changes observed in the VN were due to the hypersensitivity of TNC neurons or directly caused by NTG, viral vectors containing CGRP RNAi were injected into the trigeminal ganglion to knockdown CGRP synthesis in NTG-treated animals. We observed that c-fos expression was significantly decreased in the TNC among CM group, and similar pattern was also observed in the VN. Above data indicated the possibility of trigeminal-mediated sensitization of vestibular nucleus neurons in migraine.

CGRP is a 37-amino acid neuropeptide, and widely expressed in central and peripheral nervous system, with prominent localization in the outer laminae of the spinal 
cord dorsal horn and TNC $[15,54]$. The majority of CGRP in the TNC is synthesized in small- and mediumdiameter neurons of trigeminal ganglion, and transported to central terminals [15]. CGRP is believed to be a key regulator in central sensitization of trigeminovascular neurons, attributing to migraine headache and associated hypersensitivity $[6,15]$. Contrast to a nociceptive role, CGRP enhances the abnormal pain sensitivity rather than normal acute nociceptive signals, as evidenced by the fact that intrathecal administration of CGRP did not alter response thresholds to noxious thermal stimuli in normal rats, while anti-CGRP antiserum significantly blocked hyperalgesia under inflammatory condition [55]. Previous study demonstrates that the CGRP protein is expressed in afferent terminals in the TNC [15], and in the vestibular nuclei and cerebellum, CGRP immunoreactivity can be detected on neuronal somas $[54,56]$. In consistent with previous study, we found the extensive expression of CGRP immunoreactive fibres in the superlayer of the TNC and CGRPpositive neurons in the $\mathrm{VN}[37,56]$. To date, little evidence shows the change of CGRP expression in the VN after CM. In this study, we noted that CGRP expression was significantly elevated in the $\mathrm{VN}$ after $\mathrm{CM}$, suggesting that the endogenous CGRP expression change in the VN was in response to CM. We also found that CGRP positively stained neurons could be detected in four major vestibular nuclei after $\mathrm{CM}$, with a slight more expression in medial vestibular nucleus according to the rat brain atlas of Paxinos and Watson [56]. The medial vestibular nucleus is the largest nucleus within the vestibular complex and has various types of neurons [57]. Medial vestibular nucleus neurons are essential to the maintenance of vestibulo-ocular reflex, especially in stabilizing the images during head movement [58]. Clinical-based studies provide supporting evidence that ocular and perceptual thresholds are significantly increased in migraine patients with vestibular symptoms relative to those without vestibular symptoms, implying that sensitization of the medial vestibular nucleus might be a primary pathophysiological process underlying the vestibular hypersensitivity in migraine patients with vestibular symptoms $[10,11]$.

Clinical trials investigating CGRP antibodies or CGRP receptor antagonists have shown statistically significantly efficacy for migraine treatment [45]. Both of CGRP antibodies or CGRP receptor antagonists have limited permeability of blood-brain barrier [59], indicating that trigeminal ganglion may be the target region for antiCGRP therapy in migraine headache [59]. Present study used lentiviral vectors comprising CGRP short hairpin RNA (LV-CGRP) to knockdown CGRP production in the trigeminal ganglion. The neuronal activation and CGRP expression in the TNC were decreased, meanwhile, the migraine-associated hyperalgesia and spontaneous facial pain were alleviated after $\mathrm{CM}$, suggesting knockdown of CGRP in the trigeminal ganglion could efficiently block development of sensitization of the TNC and relieve migraine headache after CM. We also found that knockdown of CGRP in the trigeminal ganglion had similar effects on the $\mathrm{VN}$, and restored vestibular dysfunction after CM. Currently, the relationship between CGRP and sensitization of VN neurons remains obscure. Indirect evidence shows that CGRP expression is significantly increased among $\mathrm{VN}$ neurons in a preclinical model of motion sickness, whilst anisodamine could decrease CGRP expression in the VN and relieve motion sickness [17]. Collectively, these data pointed a role of CGRP in the VN to facilitate sensitization of VN neurons, and blocking CGRP in the trigeminal ganglion might relieve vestibular symptoms in migraine.

In this study, we focused on c-fos and CGRP expression changes in the TNC and VN, as well as the behavior changes after CM or CGRP knockdown. Previous studies showed that the indicators of central sensitization also contained electrophysiologic, like spontaneous and evoked activity of wide-dynamic-range neurons or response thresholds [15]. Hence, whether $\mathrm{CM}$ could induce electrophysiological changes in the $\mathrm{VN}$ neurons remains to be determined. Moreover, immunohistochemistry study reported that CGRP could be detected in various brain regions, including cerebral cortex and thalamic nuclei [54], thus, the effects of other brain regions on the regulation of neuronal activation and CGRP expression in the $\mathrm{VN}$ warrant further investigation.

\section{Conclusions}

In conclusion, we demonstrated the sensitization of vestibular nucleus neurons in a preclinical model of CM, and down-regulation of CGRP after CM could improve vestibular dysfunction, suppress neuronal activation, and reduce CGRP expression in the VN. Therefore, antiCGRP might be a promising treatment strategy for ameliorating vestibular dysfunction in migraine patients with vestibular symptoms.

\section{Abbreviations}

CGRP: Calcitonin gene-related peptide; CM: Chronic migraine; FG: Fluorogold; FOV: Field of view; GFP: Green fluorescent protein; LV-CGRP: Lentiviral vectors comprising CGRP short hairpin RNA; LV-NC: Lentivirus vector negative control; NTG: Nitroglycerin; OD: Optical density; TNC: Trigeminal nucleus caudalis; VEH: Vehicle; VM: Vestibular migraine

\section{Acknowledgements}

Not applicable.

Authors' contributions

YZ, YXZ and JZ designed the study. YZ, KT, YW, XF, QP, GQ, DZ and LC collected and analyzed the data. $Y Z$ and $Y X Z$ drafted the manuscript. Critically revising the article was done by $Y Z, Y W, X F, Q P$ and JZ. All authors read and approved the final manuscript. 


\section{Funding}

This study was supported by the National Natural Science Foundation of China [NO. 81971063] and Cultivation Fund of The First Affiliated Hospital of Chongqing Medical University [NO. PYJJ2019-20].

\section{Availability of data and materials}

The data used and analyzed in this article are available from on reasonable request.

\section{Ethics approval and consent to participate}

All animal experiments performed in this study were approved by the Ethics Committee for Animal Experimentation of Chongqing Medical University.

\section{Consent for publication}

Not applicable.

\section{Competing interests}

The authors declare that they have no conflict of interest.

\section{Author details}

'Department of Neurology, The First Affiliated Hospital of Chongqing Medical University, 1st Youyi Road, Yuzhong District, Chongqing 400016, China. ${ }^{2}$ Department of Vascular Surgery, The First Affiliated Hospital of Chongqing Medical University, Chongqing, China. ${ }^{3}$ Laboratory Research Center, The First Affiliated Hospital of Chongqing Medical University, Chongqing, China.

\section{Received: 10 March 2020 Accepted: 1 June 2020}

\section{Published online: 10 June 2020}

\section{References}

1. Collaborators GBDH (2018) Global, regional, and national burden of migraine and tension-type headache, 1990-2016: a systematic analysis for the global burden of disease study 2016. Lancet Neurol 17(11):954-976

2. Pan Q, Zhang Y, Long T, He W, Zhang S, Fan Y et al (2018) Diagnosis of Vertigo and dizziness syndromes in a neurological outpatient clinic. Eur Neurol 79(5-6):287-294

3. Carvalho GF, Vianna-Bell FH, Florencio LL, Pinheiro CF, Dach F, Bigal ME et al (2019) Presence of vestibular symptoms and related disability in migraine with and without aura and chronic migraine. Cephalalgia. 39(1):29-37

4. Headache Classification Committee of the International Headache Society (IHS) (2018) The International Classification of Headache Disorders, 3rd edition. Cephalalgia 38(1):1-211

5. Huang TC, Wang SJ, Kheradmand A (2020) Vestibular migraine: an update on current understanding and future directions. Cephalalgia. 40(1):107-12

6. Goadsby PJ, Holland PR, Martins-Oliveira M, Hoffmann J, Schankin C Akerman S (2017) Pathophysiology of migraine: a disorder of sensory processing. Physiol Rev 97(2):553-622

7. Burstein R, Noseda R, Borsook D (2015) Migraine: multiple processes, complex pathophysiology. J Neurosci 35(17):6619-6629

8. Z Zhang Y, Kong Q, Chen J, Li L, Wang D, Zhou J (2016) International classification of headache disorders 3rd edition beta-based field testing of vestibular migraine in China: demographic, clinical characteristics, audiometric findings and diagnosis statues. Cephalalgia. 36(3):240-248

9. Radtke A, Neuhauser H, von Brevern M, Hottenrott T, Lempert T (2011) Vestibular migraine--validity of clinical diagnostic criteria. Cephalalgia. 31(8): 906-913

10. Bednarczuk NF, Bonsu A, Ortega MC, Fluri AS, Chan J, Rust H et al (2019) Abnormal visuo-vestibular interactions in vestibular migraine: a cross sectional study. Brain. 142(3):606-616

11. King S, Priesol AJ, Davidi SE, Merfeld DM, Ehtemam F, Lewis RF (2019) Selfmotion perception is sensitized in vestibular migraine: pathophysiologic and clinical implications. Sci Rep 9(1):14323

12. Diagne M, Valla J, Delfini C, Buisseret-Delmas C, Buisseret P (2006) Trigeminovestibular and trigeminospinal pathways in rats: retrograde tracing compared with glutamic acid decarboxylase and glutamate immunohistochemistry. J Comp Neurol 496(6):759-772

13. Marano E, Marcelli V, Di Stasio E, Bonuso S, Vacca G, Manganelli F et al (2005) Trigeminal stimulation elicits a peripheral vestibular imbalance in migraine patients. Headache. 45(4):325-331
14. Lassen LH, Haderslev PA, Jacobsen VB, Iversen HK, Sperling B, Olesen J (2002) CGRP may play a causative role in migraine. Cephalalgia. 22(1):54-61

15. lyengar S, Ossipov MH, Johnson KW (2017) The role of calcitonin generelated peptide in peripheral and central pain mechanisms including migraine. Pain. 158(4):543-559

16. He W, Long T, Pan Q, Zhang S, Zhang Y, Zhang D et al (2019) Microglial NLRP3 inflammasome activation mediates IL-1 beta release and contributes to central sensitization in a recurrent nitroglycerin-induced migraine model. J Neuroinflammation 16(1):78

17. Xiaocheng W, Zhaohui S, Junhui X, Lei Z, Lining F, Zuoming Z (2012) Expression of calcitonin gene-related peptide in efferent vestibular system and vestibular nucleus in rats with motion sickness. PLoS One 7(10):e47308

18. Greco R, Demartini C, Zanaboni AM, Tumelero E, Reggiani A, Misto A, Piomelli D, Tassorelli C (2020 Feb) FAAH inhibition as a preventive treatment for migraine: a pre-clinical study. Neurobiol Dis 134:104624

19. Gaboyard-Niay S, Travo C, Saleur A, Broussy A, Brugeaud A, Chabbert C (2016) Correlation between afferent rearrangements and behavioral deficits after local excitotoxic insult in the mammalian vestibule: a rat model of vertigo symptoms. Dis Model Mech 9(10):1181-1192

20. Brugeaud A, Travo C, Dememes D, Lenoir M, Llorens J, Puel JL et al (2007) Control of hair cell excitability by vestibular primary sensory neurons. J Neurosci 27(13):3503-3511

21. Zhou X, Liang J, Wang J, Fei Z, Qin G, Zhang D et al (2019) Up-regulation of astrocyte excitatory amino acid transporter 2 alleviates central sensitization in a rat model of chronic migraine. J Neurochem. 2019:e14944. https://doi. org/10.1111/jnc.14944

22. Holstein GR, Friedrich VL Jr, Martinelli GP (2014) Projection neurons of the vestibulo-sympathetic reflex pathway. J Comp Neurol 522(9):2053-2074

23. Abdallah K, Artola A, Monconduit L, Dallel R, Luccarini P (2013) Bilateral descending hypothalamic projections to the spinal trigeminal nucleus caudalis in rats. PLoS One 8(8):e73022

24. Smith JB, Watson GD, Alloway KD, Schwarz C, Chakrabarti S (2015) Corticofugal projection patterns of whisker sensorimotor cortex to the sensory trigeminal nuclei. Front Neural Circuits 9:53

25. Long H, Liao L, Zhou Y, Shan D, Gao M, Huang R et al (2017) A novel technique of delivering viral vectors to trigeminal ganglia in rats. Eur J Oral Sci 125(1):1-7

26. Al Deeb S, Al Moutaery K, Khan HA, Tariq M (2000) Exacerbation of iminodipropionitrile-induced behavioral toxicity, oxidative stress, and vestibular hair cell degeneration by gentamicin in rats. Neurotoxicol Teratol 22(2):213-220

27. Ahmad Khan H, Al Deeb S, Al Moutaery K, Tariq M (2004) Metoclopramide attenuates iminodipropionitrile-induced oxidative stress and neurobehavioral toxicity in rats. Pharmacol Biochem Behav 79(3):555-561

28. Saldana-Ruiz S, Soler-Martin C, Llorens J (2012) Role of CYP2E1-mediated metabolism in the acute and vestibular toxicities of nineteen nitriles in the mouse. Toxicol Lett 208(2):125-132

29. Chanda ML, Tuttle AH, Baran I, Atlin C, Guindi D, Hathaway G et al (2013) Behavioral evidence for photophobia and stress-related ipsilateral head pain in transgenic Cacna1a mutant mice. Pain. 154(8):1254-1262

30. Zhang J, Li B, Yu L, He YC, Li HZ, Zhu JN et al (2011) A role for orexin in central vestibular motor control. Neuron. 69(4):793-804

31. Tung W, Burton TJ, Dababneh E, Quail SL, Camp AJ (2014) Behavioral assessment of the aging mouse vestibular system. J Vis Exp 89:e51605

32. Hargreaves K, Dubner R, Brown F, Flores C, Joris J (1988) A new and sensitive method for measuring thermal nociception in cutaneous hyperalgesia. Pain. 32(1):77-88

33. Mahmoudi J, Mohaddes G, Erfani M, Sadigh-Eteghad S, Karimi P, Rajabi M et al (2018) Cerebrolysin attenuates hyperalgesia, photophobia, and neuroinflammation in a nitroglycerin-induced migraine model in rats. Brain Res Bull 140:197-204

34. West MJ, Slomianka L, Gundersen HJ (1991) Unbiased stereological estimation of the total number of neurons in thesubdivisions of the rat hippocampus using the optical fractionator. Anat Rec 231(4):482-497

35. Dutheil S, Brezun JM, Leonard J, Lacour M, Tighilet B (2009) Neurogenesis and astrogenesis contribution to recovery of vestibular functions in the adult cat following unilateral vestibular neurectomy: cellular and behavioral evidence. Neuroscience. 164(4):1444-1456

36. Wang J, Fei Z, Liang J, Zhou X, Qin G, Zhang D et al (2020) EphrinB/EphB signaling contributes to the Synaptic plasticity of chronic migraine through NR2B phosphorylation. Neuroscience. 428:178-191 
37. Jing F, Zhang Y, Long T, He W, Qin G, Zhang D et al (2019) P2Y12 receptor mediates microglial activation via RhoA/ROCK pathway in the trigeminal nucleus caudalis in a mouse model of chronic migraine. J Neuroinflammation 16(1):217

38. Radtke A, von Brevern M, Neuhauser H, Hottenrott T, Lempert T (2012) Vestibular migraine: long-term follow-up of clinical symptoms and vestibulo-cochlear findings. Neurology. 79(15):1607-1614

39. Furman JM, Marcus DA, Balaban CD (2003) Migrainous vertigo: development of a pathogenetic model and structured diagnostic interview. Curr Opin Neurol 16(1):5-13

40. Kaalberg JW, Hornbuckle GC (1976) Plaque: the primary etiologic agent in dental disease--a review of the research. lowa Dent J 62(1):37-40

41. Koo JW, Balaban CD (2006) Serotonin-induced plasma extravasation in the murine inner ear: possible mechanism of migraine-associated inner ear dysfunction. Cephalalgia. 26(11):1310-1319

42. Kang WS, Lee SH, Yang CJ, Ahn JH, Chung JW, Park HJ (2016) Vestibular function tests for vestibular migraine: clinical implication of video head impulse and caloric tests. Front Neurol 7:166

43. Bates EA, Nikai T, Brennan KC, Fu YH, Charles AC, Basbaum Al et al (2010) Sumatriptan alleviates nitroglycerin-induced mechanical and thermal allodynia in mice. Cephalalgia. 30(2):170-178

44. Holland PR, Saengjaroentham C, Vila-Pueyo M (2019) The role of the brainstem in migraine: potential brainstem effects of CGRP and CGRP receptor activation in animal models. Cephalalgia. 39(3):390-402

45. Charles A, Pozo-Rosich P (2019) Targeting calcitonin gene-related peptide: a new era in migraine therapy. Lancet. 394(10210):1765-1774

46. Gurkov R, Kantner C, Strupp M, Flatz W, Krause E, Ertl-Wagner B (2014) Endolymphatic hydrops in patients with vestibular migraine and auditory symptoms. Eur Arch Otorhinolaryngol 271(10):2661-2667

47. Arslan Y, Arslan IB, Aydin H, Yagiz O, Tokucoglu F, Cukurova I (2017) The etiological relationship between migraine and sudden hearing loss. Otol Neurotol 38(10):1411-1414

48. de Tommaso M, Ambrosini A, Brighina F, Coppola G, Perrotta A, Pierelli F et al (2014) Altered processing of sensory stimuli in patients with migraine. Nat Rev Neurol 10(3):144-155

49. Woolf CJ (2011) Central sensitization: implications for the diagnosis and treatment of pain. Pain 152(3 Suppl):S2-S15

50. Jeong SH, Oh SY, Kim HJ, Koo JW, Kim JS (2010) Vestibular dysfunction in migraine: effects of associated vertigo and motion sickness. J Neurol 257(6): 905-912

51. Oshinsky ML (2014) Sensitization and ongoing activation in the trigeminal nucleus caudalis. Pain. 155(7):1181-1182

52. Boyer N, Dallel R, Artola A, Monconduit L (2014) General trigeminospinal central sensitization and impaired descending pain inhibitory controls contribute to migraine progression. Pain. 155(7):1196-1205

53. Murray AJ, Croce K, Belton T, Akay T, Jessell TM (2018) Balance control mediated by vestibular circuits directing limb extension or antagonist muscle co-activation. Cell Rep 22(5):1325-1338

54. Warfvinge K, Edvinsson L (2019) Distribution of CGRP and CGRP receptor components in the rat brain. Cephalalgia. 39(3):342-353

55. Kawamura M, Kuraishi Y, Minami M, Satoh M (1989) Antinociceptive effect of intrathecally administered antiserum against calcitonin gene-related peptide on thermal and mechanical noxious stimuli in experimental hyperalgesic rats. Brain Res 497(1):199-203

56. Ahn SK, Khalmuratova R, Jeon SY, Kim JP, Park JJ, Hur DG et al (2009) Colocalization of 5-HT1F receptor and calcitonin gene-related peptide in rat vestibular nuclei. Neurosci Lett 465(2):151-156

57. Alvarez JC, Diaz C, Suarez C, Fernandez JA (1998) Gonzalez del Rey C, Navarro $\mathrm{a}$, et al. neuronal loss in human medial vestibular nucleus. Anat Rec 251(4):431-438

58. Li YH, Li Y, Zheng L, Wang J (2020) Postsynaptic GluN2B-containing NMDA receptors contribute to long-term depression induction in medial vestibular nucleus neurons of juvenile rats. Neurosci Lett 715:134674

59. Edvinsson L, Warfvinge K (2019) Recognizing the role of CGRP and CGRP receptors in migraine and its treatment. Cephalalgia. 39(3):366-373

\section{Publisher's Note}

Springer Nature remains neutral with regard to jurisdictional claims in published maps and institutional affiliations.

Ready to submit your research? Choose BMC and benefit from:

- fast, convenient online submission

- thorough peer review by experienced researchers in your field

- rapid publication on acceptance

- support for research data, including large and complex data types

- gold Open Access which fosters wider collaboration and increased citations

- maximum visibility for your research: over $100 \mathrm{M}$ website views per year

At BMC, research is always in progress.

Learn more biomedcentral.com/submissions 